\title{
Alcohol and survival in ESCC: Prediagnosis alcohol consumption and postoperative survival in lymph node-negative esophageal carcinoma patients
}

\author{
Qilong Ma ${ }^{1, *}$, Wengao Liu ${ }^{1, *}$, Ran Jia ${ }^{1, *}$, Hao Long ${ }^{1}$, Lanjun Zhang ${ }^{1}$, Peng Lin ${ }^{1}$, \\ Hongyun Zhao $^{1}$ and Guowei Ma $^{1}$ \\ ${ }^{1}$ Sun Yat-sen University Cancer Center, Guangdong Esophageal Cancer Institute, State Key Laboratory of Oncology in South \\ China, Collaborative Innovation Center for Cancer Medicine, Guangzhou, China \\ * These authors have contributed equally to this work \\ Correspondence to: Hongyun Zhao, email: zhaohy@sysucc.org.cn
}

Guowei Ma, email: magw@sysucc.org.cn

Keywords: alcohol consumption, postoperative survival, esophageal carcinoma, Chinese cohort Received: October 11,2015 Accepted: March 31,2016 Published: April 15, 2016

\section{ABSTRACT}

Background: The association between esophageal cancer and prediagnosis alcohol consumption is well established. However, evidence that prediagnosis alcohol consumption affects postoperative survival in patients with lymph node-negative esophageal squamous cell carcinoma (ESCC) is lacking. We conducted a retrospective study on the effect of prediagnosis alcohol consumption on the postoperative survival of patients with lymph node-negative ESCC in China.

Methods: We enrolled 643 ESCC patients with negative lymphatic metastasis who had undergone esophagectomy between 1990 and 2005 at the Department of Thoracic Surgery, Sun Yat-sen University Cancer Center, Guangzhou, China, and reviewed their demographic, pathologic, preoperative, and cancer outcome data obtained from medical records. These data were analyzed using life table and Kaplan-Meier analyses and multivariate Cox regression.

Results: There was a significant reduction in 3- and 5-year survival in drinkers with lymph node-negative ESCC. For drinkers, 3- and 5-year survival rates were $43 \%$ and $36 \%$ respectively, whereas, for nondrinkers, the corresponding values were $63 \%$ and $58 \%$, respectively $(p<0.05)$. Multivariate Cox regression showed that drinking $(p=0.001$, relative risk $=1.583)$ was an independent factor for survival in patients with lymph node-negative ESCC. Striated analysis revealed that drinking was an independent factor for survival in patients with stage II A $(p=0.008$, relative risk $=1.679)$, stage IB $(p=0.044$, relative risk $=1.517)$, and well $(p=0.011$, relative risk $=1.783)$ and moderately $(p=0.002$, relative risk $=1.915)$ differentiated ESCC.

Conclusions: Prediagnosis alcohol consumption is an independent prognostic factor for postoperative survival in patients with lymph node-negative ESCC.

\section{INTRODUCTION}

Esophageal cancer is one of the most common malignant tumors, ranking sixth in the causes of cancer mortality worldwide [1]. It is prevalent in China, Iran, South Africa, Uruguay, France, and Italy, of which China has almost half the total cases and the highest mortality rate [2]. In China, esophageal cancer is primarily of the squamous cell type, which accounts for $>95 \%$ cases [3]. Despite improvements in nonsurgical treatment, surgery remains the mainstay of curative treatments. The outcome of surgical resection for esophageal cancer is poor. Although the postoperative 5-year survival rate of esophageal cancer is just $20 \%-40 \%$ in China [4], it is still higher than that reported in Western countries [5].

Numerous studies on esophageal cancer etiology 
have been conducted over the past decade. Smoking and prediagnosis alcohol consumption are well-known risk factors [6-9]. Many factors have been identified as prognostic, including tumor biological behavior, postoperative treatment, operative technique, and response to preoperative chemoradiotherapy [10]. Alcohol consumption is rising worldwide, and, according to a recent World Cancer Research Fund report [11], is a convincing risk factor for esophageal cancer. Given the role of alcohol consumption in the etiology of esophageal cancer, it is reasonable to hypothesize that it also influences tumor progression and patient survival.

We report the findings of a large retrospective study in a cohort of patients with lymph node-negative esophageal squamous cell carcinoma (ESCC), where in the influence of prediagnosis alcohol consumption on postoperative survival in patients with different grades and stages of ESCC, as well as the mechanism underlying these effects was investigated.

\section{MATERIALS AND METHODS}

We performed a large retrospective patient analysis by searching the esophageal cancer database of the Department of Thoracic Surgery at Sun Yat-sen University Cancer Center, Guangzhou, China. We enrolled 643 patients with negative lymphatic metastasis who had undergone esophagectomy between 1990 and 2005 at this institute. Patients were not eligible if tumors were located at the cervical esophagus or esophagogastric junction, or had other histological subtypes of esophageal cancer besides ESCC. None of the patients died as postoperative complication, and none received preoperative chemotherapy or irradiation. Sun Yat-sen University Cancer Center Hospital Ethics Committee approved the study. Mean follow-up duration was 6.5 years (range: 1-20 years).

Clinical and pathological data were extracted from medical records. Baseline data included age, sex, smoking, alcohol consumption, family history, surgical technique, and tumor biological features. Tumors were staged according to the American Joint Committee on Cancer (AJCC) Staging Manual ( $6^{\text {th }}$ Edition). Patients with a present or past history of alcohol consumption were referred to as drinkers.

Analyses were conducted using SPSS version 18.0 statistical software (IBM SPSS, Inc., Chicago, IL, USA). Descriptive statistics (e.g. frequency, mean, standard deviation) were obtained for demographic, epidemiologic, and clinical patient characteristics. Life table analysis was used to calculate the 3- and 5-year survival rates of patients, whereas Kaplan-Meier analysis was used to calculate their survival probability. Survival curves were generated according to drinking history (Figure 1), and the log rank test was used to determine the statistical significance of differences between the survival curves of drinkers and those of nondrinkers. Stage-stratified analysis with the logrank test was used to study the influence of alcohol consumption on cancer at different stages. A $p$-value of $<0.05$ was considered statistically significant. Multivariate Cox regression was used to exclude other confounding factors affecting survival. Survival was defined as time from date of surgery to date of death, with living patients censored at the date of last follow-up or date of analysis.

\section{RESULTS}

\section{Patient groups according to drinking history}

Patients were categorized as drinkers or nondrinkers on the basis of their drinking history. Table 1 summarizes patient demographics. There were 121 (18.8\%) drinkers and $522(81.2 \%)$ nondrinkers. Drinkers were more commonly male $(118 ; 97.5 \% ; P<0.05)$, and most of them had a smoking history as well $(111 ; 91.7 \% ; P<$ $0.05)$. Tumor location was significantly different $(p=$ 0.006 ) between drinkers and nondrinkers. No significant differences in age, family history, tumor features, surgical technique, or postoperative treatment were evident between the two groups.

According to the results of life table analysis, the 3and 5 -year survival rates were $43 \%$ and $36 \%$, respectively, in drinkers and $63 \%$ and $58 \%$, respectively, in nondrinkers. According to the results of Kaplan-Meier analysis and the logrank test, overall survival duration was significantly longer in nondrinkers than in drinkers $(p<0.05)$.

The results of multivariate Cox regression (Table 2) indicated that drinking [relative risk $(\mathrm{RR})=1.583, p$ $=0.001]$, surgical technique $(\mathrm{RR}=1.107, p=0.023)$, postoperative staging $(\mathrm{RR}=1.332, P=0.002)$, and tumor grade $(\mathrm{RR}=1.182, p=0.027)$ were independent prognostic factors for survival in patients with ESCC.

\section{Drinking and postoperative staging}

The results of stage-stratified analysis are demonstrated in Figure 2 and Table 3. Results of the logrank test indicated that drinking had a significant influence on survival in patients with stage IB $(p=0.015)$ and stage IIA $(p<0.01)$ ESCC, with no effect in patients with stage IA $(p=0.190)$ ESCC. In addition, multivariate analysis (Table 3 ), which excluded confounding factors, showed that drinking was an independent factor for survival in patients with Stage IIA $(p=0.008 ; \mathrm{RR}=1.679)$ and stage IB ( $p=0.044 ; \mathrm{RR}=1.517) \mathrm{ESCC}$, with no effect in patients with Stage IA ESCC. 
Table 1: Baseline characteristic grouped by history of drinking

\begin{tabular}{|c|c|c|c|}
\hline & drinker & non-drinker & $P$-value \\
\hline gender & & & $<0.05$ \\
\hline Male & $118(97.5 \%)$ & $336(64.4 \%)$ & \\
\hline Female & $3(2.5 \%)$ & $186(35.6 \%)$ & \\
\hline age & & & 0.275 \\
\hline$<40$ & $3(2.5 \%)$ & $30(5.7 \%)$ & \\
\hline $40 \sim 60$ & $80(66.1 \%)$ & $318(60.9 \%)$ & \\
\hline$>60$ & $38(31.4 \%)$ & $174(33.3 \%)$ & \\
\hline Smoker & $111(91.7 \%)$ & $278(53.3 \%)$ & $<0.05$ \\
\hline family history & $8(6.6 \%)$ & $38(7.3 \%)$ & 0.797 \\
\hline Tumor location & & & 0.006 \\
\hline upper thoracic & $17(14 \%)$ & $54(10.3 \%)$ & \\
\hline middle thoracic & $73(60.3 \%)$ & $389(74.5 \%)$ & \\
\hline lower thoracic & $31(25.6 \%)$ & $79(15.1 \%)$ & \\
\hline post-operative stage & & & 0.777 \\
\hline IA & $8(6.6 \%)$ & $40(7.7 \%)$ & \\
\hline IB & $55(45.5 \%)$ & $249(47.7 \%)$ & \\
\hline IIA & $58(47.9 \%)$ & $233(44.6 \%)$ & \\
\hline tumor grade & & & 0.229 \\
\hline well & $43(35.5 \%)$ & $198(37.9 \%)$ & \\
\hline moderately & $58(47.9 \%)$ & $209(40.1 \%)$ & \\
\hline poorly & $20(16.5 \%)$ & $114(22 \%)$ & \\
\hline surgery incision & & & 0.628 \\
\hline right thoracic & $26(21.5 \%)$ & $118(22.6 \%)$ & \\
\hline Left thoracic & $95(78.5 \%)$ & $404(77.4 \%)$ & \\
\hline
\end{tabular}

*1 value of tumor grade is missing.

Table 2: Multivariable analysis of factors related to ESCC survival

\begin{tabular}{|l|l|l|l|l|}
\hline & & & \multicolumn{2}{|l|}{$95.0 \%$ CI for Exp(B) } \\
\hline & $\boldsymbol{P}$ & HR & Lower & Upper \\
\hline gender & .422 & .859 & .592 & 1.245 \\
\hline Age & .887 & 1.015 & .830 & 1.241 \\
\hline smoking & .153 & 1.280 & .913 & 1.794 \\
\hline drinking & .001 & 1.583 & 1.213 & 2.074 \\
\hline Family history & .388 & .814 & .511 & 1.298 \\
\hline Tumor location & .703 & .958 & .770 & 1.193 \\
\hline Surgery technique & .023 & 1.107 & 1.014 & 1.209 \\
\hline Post-operative staging & .002 & 1.332 & 1.108 & 1.602 \\
\hline Tumor grade & .027 & 1.182 & 1.019 & 1.372 \\
\hline
\end{tabular}


Table 3: Multivariable analysis of factors related to ESCC survival grouped by staging

\begin{tabular}{|l|l|l|l|l|l|l|}
\hline \multicolumn{2}{|l}{ IA } & RR & $\boldsymbol{P}$ & RR & $\boldsymbol{P}$ & RR \\
\hline & $\boldsymbol{P}$ & RR & \multicolumn{2}{l}{ IIA } \\
\hline gender & 0.644 & 0.567 & 0.369 & 1.285 & 0.024 & 0.532 \\
\hline age & 0.089 & 0.376 & 0.786 & 1.041 & 0.398 & 1.138 \\
\hline smoking & 0.316 & 3.335 & 0.126 & 1.480 & 0.819 & 0.946 \\
\hline drinking & 0.084 & 4.224 & 0.044 & 1.517 & 0.008 & 1.679 \\
\hline Family history & 0.077 & 0.104 & 0.199 & 0.624 & 0.791 & 1.092 \\
\hline location & 0.082 & 0.247 & 0.704 & 0.942 & 0.484 & 1.126 \\
\hline surgery & 0.140 & 1.482 & 0.113 & 1.111 & 0.215 & 1.086 \\
\hline $\begin{array}{l}\text { Differentiation } \\
\text { grade }\end{array}$ & 0.021 & 3.206 & 0.035 & 1.266 & 0.698 & 1.044 \\
\hline
\end{tabular}

Table 4: Multivariable analysis of factors related to ESCC survival grouped by differentiation grade

\begin{tabular}{|c|c|c|c|c|c|c|}
\hline & \multicolumn{2}{|l|}{ well } & \multicolumn{2}{|c|}{ moderate } & \multicolumn{2}{|l|}{ poor } \\
\hline & $P$ & $\mathbf{R R}$ & $P$ & RR & $P$ & $\mathbf{R R}$ \\
\hline gender & 0.789 & 1.092 & 0.262 & 0.726 & 0.573 & 0.781 \\
\hline age & 0.999 & 1.000 & 0.857 & 0.971 & 0.620 & 1.108 \\
\hline smoking & 0.018 & 1.959 & 0.404 & 0.797 & 0.456 & 1.349 \\
\hline drinking & 0.011 & 1.783 & 0.002 & 1.915 & 0.390 & 1.308 \\
\hline Family history & 0.783 & 1.116 & 0.460 & 0.773 & 0.266 & 0.508 \\
\hline location & 0.303 & 0.821 & 0.217 & 1.254 & 0.222 & 0.754 \\
\hline surgery & 0.398 & 1.066 & 0.010 & 1.206 & 0.697 & 1.038 \\
\hline $\begin{array}{l}\text { Post-operative } \\
\text { staging }\end{array}$ & 0.014 & 1.486 & 0.025 & 1.408 & 0.922 & 1.018 \\
\hline
\end{tabular}

\section{Drinking and differential grade}

Figure 3 and Table 4 show results of survival analysis stratified by differential grade. Results of the logrank test indicated that drinking had a significant influence on survival in patients with well $(p=0.003)$ and moderately differentiated $(p=0.002)$ ESCC, with no effect in patients with poorly differentiated $\operatorname{ESCC}(p=$ 0.65 ). In addition, multivariate analysis (Table 4), which excluded confounding factors, showed that drinking was an independent factor for survival in patients with well $(P$ $=0.011, \mathrm{RR}=1.783)$ and moderately differentiated ESCC $(P=0.002, \mathrm{RR}=1.915)$, with no effect in patients with poorly differentiated ESCC

\section{CONCLUSIONS}

Esophageal cancer is currently the fifth most common and fourth most lethal malignant tumor in China. Although the incidence of esophageal adenocarcinoma is rising in Western countries, it remains unchanged in China, where ESCC accounts for the majority of esophageal cancer cases $[3,19]$.

Numerous studies have been conducted to determine the prognostic factors for ESCC survival. Various biological aspects of tumors reportedly correlate 
with prognosis. Tumor grade and stage are well-known prognostic factors, especially high tumor grade and stage [12-14]. In our study, multivariate analysis consistently identified postoperative staging $(P=0.002, \mathrm{RR}=1.332)$ and tumor grade $(p=0.027, \mathrm{RR}=1.182)$ as factors influencing survival, further confirming the role of biological factors in the survival of patients with ESCC.

Pathological information is valuable for predicting prognosis in esophageal cancer patients. However, this is not accurate enough, as prognosis can also be influenced by prediagnostic and postoperative factors. Our study focused on the influence of drinking on ESCC survival and demonstrated that drinking significantly reduces the 3- and 5-year survival rates of patients with ESCC. Multivariate Cox regression also indicates drinking as an independent factor influencing survival in these patients.
Potential mechanisms by which alcohol consumption may affect survival include increase in local permeability [15], suppression of immune function [16], and generation of metabolites that are carcinogenic to humans [17].

Stage-stratified analysis demonstrated a significant difference in survival between drinkers and nondrinkers with stage IIA or stage IB ESCC. According to analysis striated by grading, a significant difference in survival was observed between drinkers and nondrinkers with well or moderately differentiated ESCC. Possible explanations are described below. Firstly, there lack of power in the stratified datasets. There were only 8 drinkers and 40 nondrinkers with stage IA ESCC; these numbers were far from enough to display significance. The situation was similar with poorly differentiated ESCC, which was identified in 20 drinkers and 114 nondrinkers. As Figures

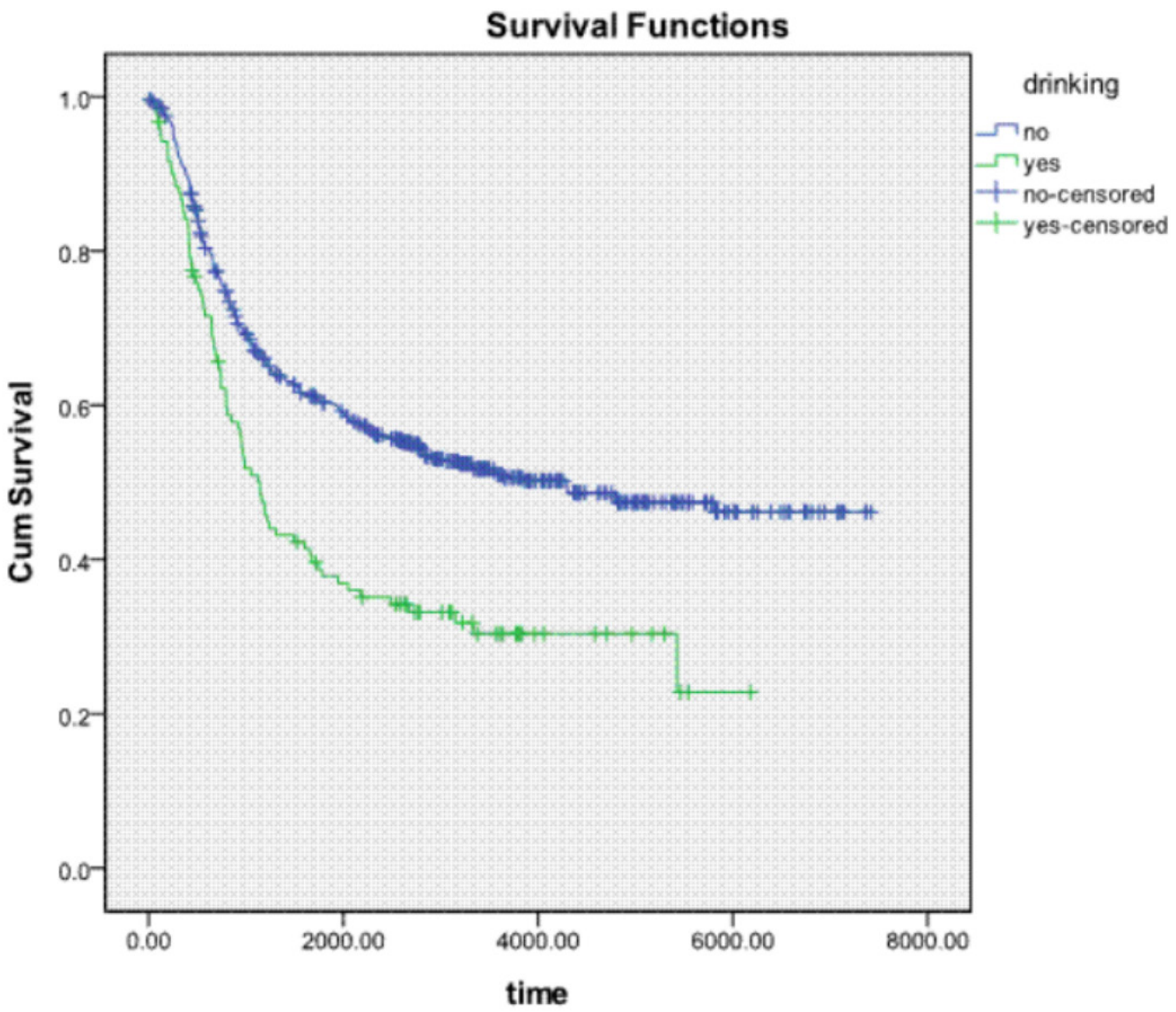

Figure 1: Kaplan-Meier survival curve of patients grouped by drinking and nondrinking habit. 
2 and 3 show, there is a trend of curve separation for Stage IA and poorly differentiated ESCC. The second possible explanation could be the different impact of drinking on tumors of different grades and stages. It is known that alcohol dehydrogenase (ADH) and aldehyde dehydrogenase play a key role in ethanol metabolism. Total ADH activity is significantly higher in cancer tissues than in healthy organs [18]. We conclude that our results can be explained by variable ADH activity in different grades and stages, leading to an enhanced accumulation of toxic acetaldehyde.

The strengths and limitations of our study should be considered while interpreting these results. Our strengths include a large sample of consecutive patients from a wellmaintained database and an efficient recording medical system containing abundant tumor information, such as tumor grade and stage. Moreover, our study showed that alcohol consumption prior to diagnosis, a well-known risk factor for cancer development, also appears to affect cancer outcome. This suggests that any drinking is a risk factor for ESCC patient survival.

Our study has all the constraints of retrospective analysis. First, a comparison of drinkers and nondrinkers is subject to selection bias. Many studies have shown that nutrition, diet, and socioeconomic factors also affect the survival of ESCC patients [20-22]. However, we failed to investigate these factors, which may have had an influence on survival in our study.

In our study, we staged tumors according to the AJCC Staging Manual ( $6^{\text {th }}$ Edition) because it was a retrospective study that enrolled patients between 1990 and 2005. According to the sixth edition, tumors should be sectioned according to tumor center location, with the carina as a reference point. Conversely, according to the new edition, tumors should be sectioned according to their upper border, with the azygos vein and the inferior pulmonary vein as reference points. Therefore, because retrospective use of the latest edition was difficult and could lead to inaccuracy, we used the sixth edition of the AJCC Staging Manual in our study.

Despite these limitations, our study identified prediagnosis alcohol consumption as an independent factor for postoperative survival in patients with lymph node-negative ESCC after adjusting for other confounding
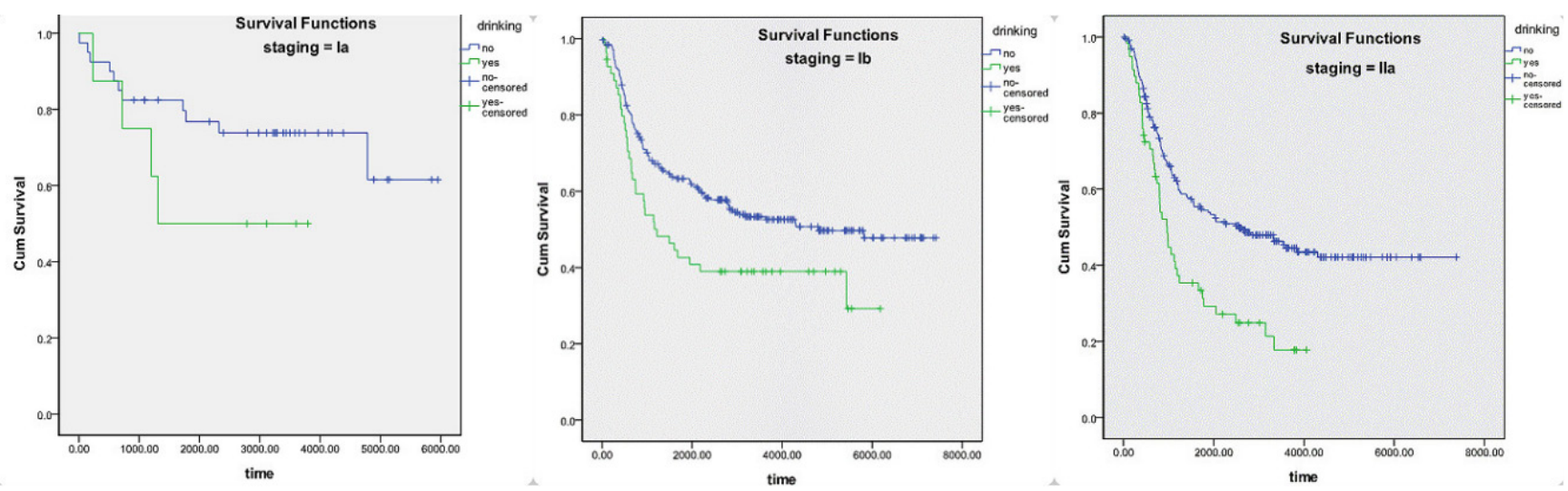

Figure 2: Kaplan-Meier survival curve striated by postoperative staging of ESCC.
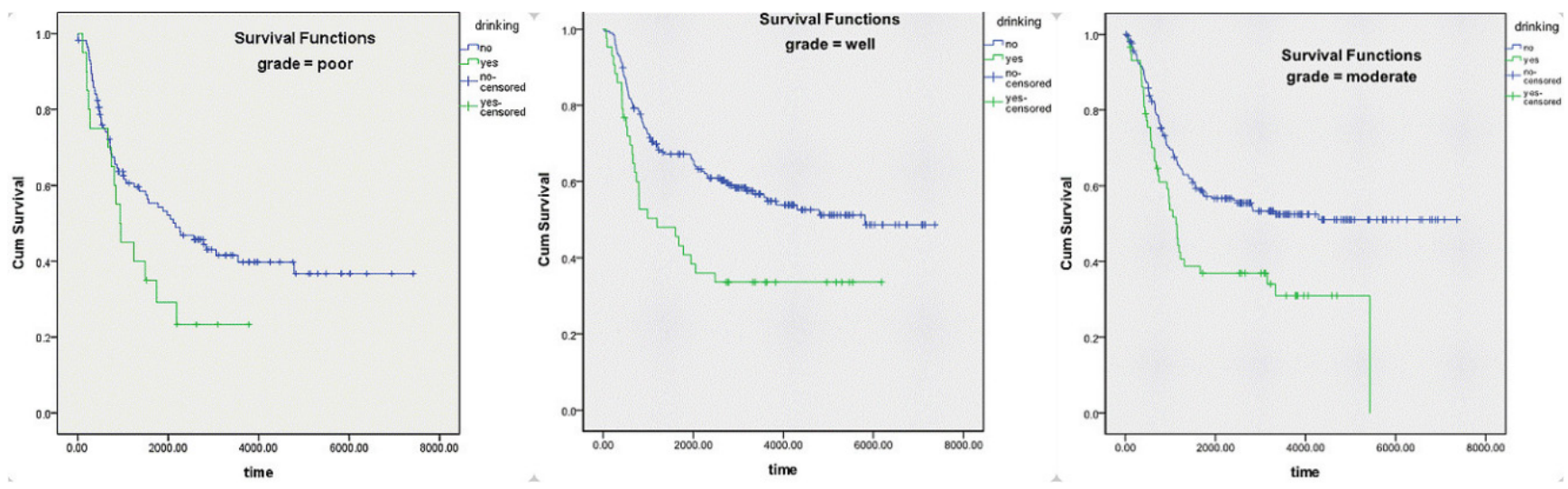

Figure 3: Kaplan-Meier survival curve striated by grade of differentiation of ESCC. 
factors, such as sex, smoking and tumor features. Decreasing the daily intake of alcohol is therefore important for preventing ESCC development or for obtaining a good prognosis in patients with ESCC.

\section{ACKNOWLEDGMENTS}

This work was supported by the Science and Technology Planning Project of Guangdong Province, China (No. 2012B031800463) and the Science and Technology Planning Project of Guangdong Province, China (No. 2013B022000040).

\section{CONFLICTS OF INTEREST}

There is no conflict of interest.

\section{REFERENCES}

1. Jemal A, Siegel R, Xu JQ, Ward E. Cancer Statistics, 2010. CA Cancer J Clin. 2010; 60:277-300.

2. Lu S, Lin P, Wang G, Luo X, Wu M. Comprehensive prevention and treatment for esophageal cancer. Chin Med J (Engl). 1999; 112:918-923.

3. Mao YS, He J, Cheng GY. Current status of surgical management of esophageal cancer in China and the future strategy. [Article in Chinese]. Zhonghua Zhong Liu Za Zhi. 2010; 32:401-404.

4. Liu JF, Wang QZ, Hou J. Surgical treatment for cancer of the esophagus and gastric cardia in Hebei, China. Br J Surg. 2004; 91:90-98.

5. Jamieson GG, Mathew G, Ludemann R, Wayman J, Myers JC, Devitt PG. Postoperative mortality following esophagectomy and problems in reporting its rate. $\mathrm{Br} \mathrm{J}$ Surg. 2004; 91:943-947.

6. Castellsagué X, Muñoz N, De Stefani E, Victora CG, Castelletto R, Rolón PA, Quintana MJ. Independent and joint effects of tobacco smoking and alcohol drinking on the risk of esophageal cancer in men and women. Int J Cancer. 1999; 82:657-664.

7. Lundell LR. Etiology and risk factors for esophageal carcinoma. Dig Dis. 2010; 28:641-644.

8. Macfarlane TV, Macfarlane GJ, Oliver RJ, Benhamou S, Bouchardy C, Ahrens W, Pohlabeln H, Lagiou P, Lagiou A, Castellsague X, Agudo A, Merletti F, Richiardi L, et al. The aetiology of upper aerodigestive tract cancers among young adults in Europe: the ARCAGE study. Cancer Causes Control. 2010; 21:2213-2221.

9. Christein JD, Hollinger EF, Millikan KW. Prognostic factors associated with resectable carcinoma of the esophagus. Am Surg. 2002; 68:258-262.

10. Kim T, Grobmyer SR, Smith R, Ben-David K, Ang D, Vogel SB, Hochwald SN. Esophageal cancer-the five year survivors. J Surg Oncol. 2011; 103:179-183.
11. Wiseman M. The second World Cancer Research Fund/ American Institute for Cancer Research expert report. Food, nutrition, physical activity, and the prevention of cancer: a global perspective. Proc Nutr Soc. 2008; 67:253-6.

12. Thrift AP, Nagle CM, Fahey PP, Russell A, Smithers BM, Watson DI, Whiteman DC; Australian Cancer Study Clinical Follow-Up Study. The influence of prediagnostic demographic and lifestyle factors on esophageal squamous cell carcinoma survival. Int J Cancer. 2012; 131:E759-68.

13. Eloubeidi MA, Desmond R, Arguedas MR, Reed CE, Wilcox CM. Prognostic factors for the survival of patients with esophageal carcinoma in the U.S.: the importance of tumor length and lymph node status. Cancer. 2002; 95:1434-1443.

14. Yoon HH, Khan M, Shi Q, Cassivi SD, Wu TT, Quevedo JF, Burch PA, Sinicrope FA, Diasio RB. The prognostic value of clinical and pathologic factors in esophageal adenocarcinoma: a mayo cohort of 796 patients with extended follow-up after surgical resection. Mayo Clin Proc. 2010; 85:1080-1089.

15. Homann N. Alcohol and upper gastrointestinal tract cancer: the role of local acetaldehyde production. Addict Biol. 2001; 6:309-323.

16. Cook RT. Alcohol abuse, alcoholism, and damage to the immune system - a review. Alcohol Clin Exp Res. 1998; 22:1927-1942.

17. Blot WJ. Alcohol and cancer. Cancer Res. 1992; 52 (7 Suppl: 2119s-2123s).

18. Jelski W, Szmitkowski M. Alcohol dehydrogenase (ADH) and aldehyde dehydrogenase (ALDH) in the cancer diseases. Clin Chim Acta. 2008; 395:1-5.

19. Shibata A, Matsuda T, Ajiki W, Sobue T. Trend in incidence of adenocarcinoma of the esophagus in Japan, 1993-2001. Jpn J Clin Oncol. 2008; 38:464-468.

20. Berretta M, Lleshi A, Fisichella R, Berretta S, Basile F, Li Volti G, Bolognese A, Biondi A, De Paoli P, Tirelli U, Cappellani A. The role of nutrition in the development of esophageal cancer: what do we know? Front Biosci (Elite Ed). 2012; 4:351-357.

21. Tian J, Chen ZC, Hang LF. The effects of nutrition status of patients with digestive system cancers on prognosis of the disease. Cancer Nurs. 2008; 31:462-467; quiz 468-469.

22. Launay L, Dejardin O, Pornet C, Morlais F, Guittet L, Launoy G, Bouvier V. Influence of socioeconomic environment on survival in patients diagnosed with esophageal cancer: a population-based study. Dis Esophagus 2012; 25:723-30. 